\title{
An STM study of molecular-beam epitaxy growth of GaAs
}

\author{
J. Sudijono, M.D. Johnson, M.B. Elowitz, C.W. Snyder and B.G. Orr \\ H.M. Randall Laboratory, University of Michigan, Ann Arbor, MI 48109-1120, USA
}

Received 7 August 1992; accepted for publication 18 September 1992

\begin{abstract}
Scanning tunneling microscopy has been used to investigate molecular-beam epitaxy growth of GaAs. By quenching the sample during deposition, we have imaged the GaAs(001) surface as it appeared during growth. Large scale inages of the surface have been obtained at coverages varying from 0.25 to 1500 layers.
\end{abstract}

\section{Introduction}

Molecular beam epitaxy (MBE) has evolved into a very powerful technique for the growth of single-crystal materials. Epitaxial structures can now be designed and fabricated with monolaycr control. Reflection high energy electron diffraction (RHEED) is the standard analytic tool used to characterize samples during MBE growth [1]. RHEED specular intensity oscillations are often used to control both the thickness and nucleation dynamics of the growing film. This technique is tremendously sensitive to surface structure. However, due to strong interactions of the scattered electrons with the surface, the diffraction data is often very difficult to interpret unambiguously. This points to a need to obtain real space images of the growth surface.

In this paper we present scanning tunneling microscopy (STM) images of GaAs surfaces as they appeared during deposition. The experiment consisted of initiating growth from a recovered surface and then terminating deposition at a specific point during the growth process. This procedure was then repeated for various termination points. Because the samples were removed from the STM for regrowth, no direct comparison can be made between any specific feature in the progression of the images. We have imaged large areas at multiple sites on multiple samples; the images shown are representative of the surface. There appears to be very little inhomogeneity in the samples.

\section{Experiment}

Deposition was performed in a standard ultrahigh vacuum system, base pressure $7 \times 10^{-11}$ Torr. Effusion cells were used to produce both the $\mathrm{Ga}$ and $\mathrm{As}_{4}$ fluxes. Commercial GaAs(001) substrates were first chemically cleaned and then loaded in the vacuum system where the oxide was completely removed at $585^{\circ} \mathrm{C}$ under an $\mathrm{As}_{4}$ flux. To smooth the substrate a $500 \mathrm{~nm}$ thick buffer layer was grown. The substrate temperature during deposition was $555^{\circ} \mathrm{C}$. The As-to-Ga pressure ratio was 15 and the deposition rate was $1 \mathrm{Ga}$ layer deposited per $6 \mathrm{~s}$. The sample miscut as determined by STM was approximately $0.15^{\circ}$ towards the (110) direction. The incident angle of the RHEED beam was approximately $0.9^{\circ}$ and corresponds closely to the "in-phase" Bragg condition. This diffraction condition was chosen as to yield a reduction in specularly scattered electron intensity with the initiation of growth [2]. The azimuthal angle was along the sample [110] direction.

A central technical point of the experiment was the ability to quench the surface morphology 
as it appeared during growth. To accomplish this a resistively heated low thermal mass sample was used. The sample temperature could be reduced from that during growth $\left(555^{\circ} \mathrm{C}\right)$ to below $450^{\circ} \mathrm{C}$ in $1.5 \mathrm{~s}$. A $\mathrm{LN}_{2}$ cooled baffle with a cooled shutter shrouds the sample [3]. The quench procedure proceeds as follows: close the $\mathrm{Ga}$ and $\mathrm{As}$ shutter simultaneously, close the $\mathrm{LN}_{2}$ cooled copper shutter, reduce the sample temperature to between 400 and $450^{\circ} \mathrm{C}$ (total elapsed time $2 \mathrm{~s}$ ), lower the temperature of the $\mathrm{Ga}$ and As sources, allow the background As pressure to drop into the low $10^{-9}$ Torr range $(\sim 20 \mathrm{~min})$, turn off the sample heating, and transfer into the STM chamber. The RHEED intensity is recorded the entire time before the transfer. We find that the RHEED intensity is quite steady during this period. Holding the sample at $400^{\circ} \mathrm{C}$ appears to eliminate physisorption of As while not allowing significant step edge motion. The surface reconstruction, as determined by and RHEED and STM, was $2 \times 4$ throughout the growth and quench [4].

\section{Results}

The data from this study consists primarily of STM topographs. In this paper we have chosen to present large-scale mosaic images. It is our hope that by viewing such data the reader will obtain a better understanding of the general GaAs(001) surface morphology and its evolution during MBE growth.

To place the sequence of images in a context, fig. 1 shows a schematic RHEED tracing for low temperature GaAs growth. Points labeled on the curve indicate deposition thicknesses studied by STM. Figs. 2-7 show the STM images corresponding to the labeled arrows of fig. 1 .

\section{Discussion}

Fig. 2 shows the starting surface for growth. It is prepared by first depositing a buffer layer and than letting the surface recover for $\sim 600 \mathrm{~s}$ at a temperature of $555^{\circ} \mathrm{C}$. This procedure produces a

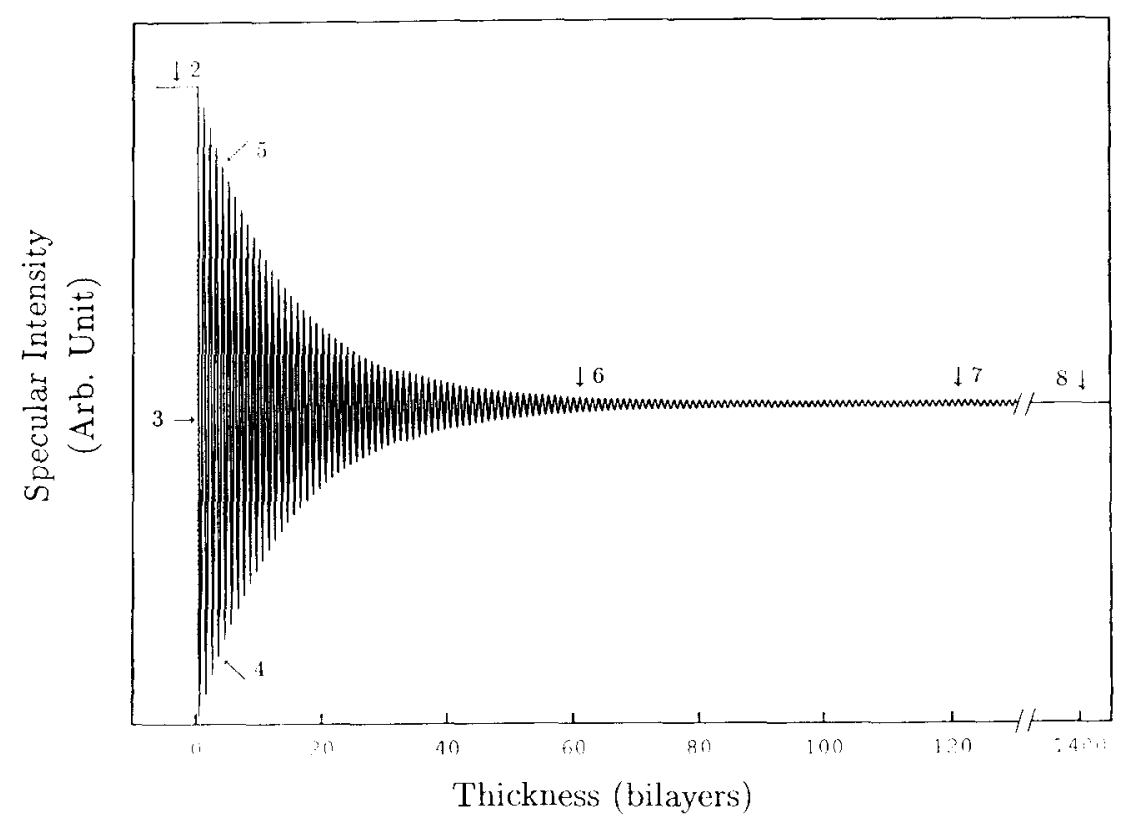

Fig. 1. Schematic RHEED intensity oscillation curve. The arrows label the points where growth was terminated and quenched. The following STM images show the GaAs surface as it appeared at each stage of growth. 
very smooth surface. Steps are often quite regularly spaced with a typical terrace width of 1500 $\AA$. From this data we estimate the macroscopic viscinality of the sample to be $0.15^{\circ}$. Occasionally, step bunching is observed on this surface [5]. Often these groups of steps appear as very large, highly anisotropic islands that run along the [110] direction. The typical height is 7 layers and the length of the major axis is greater than $2 \mu \mathrm{m}$. The upper left corner of fig. 2 shows a region of such step bunching. Due to the size of these features they are difficult to study with the STM.

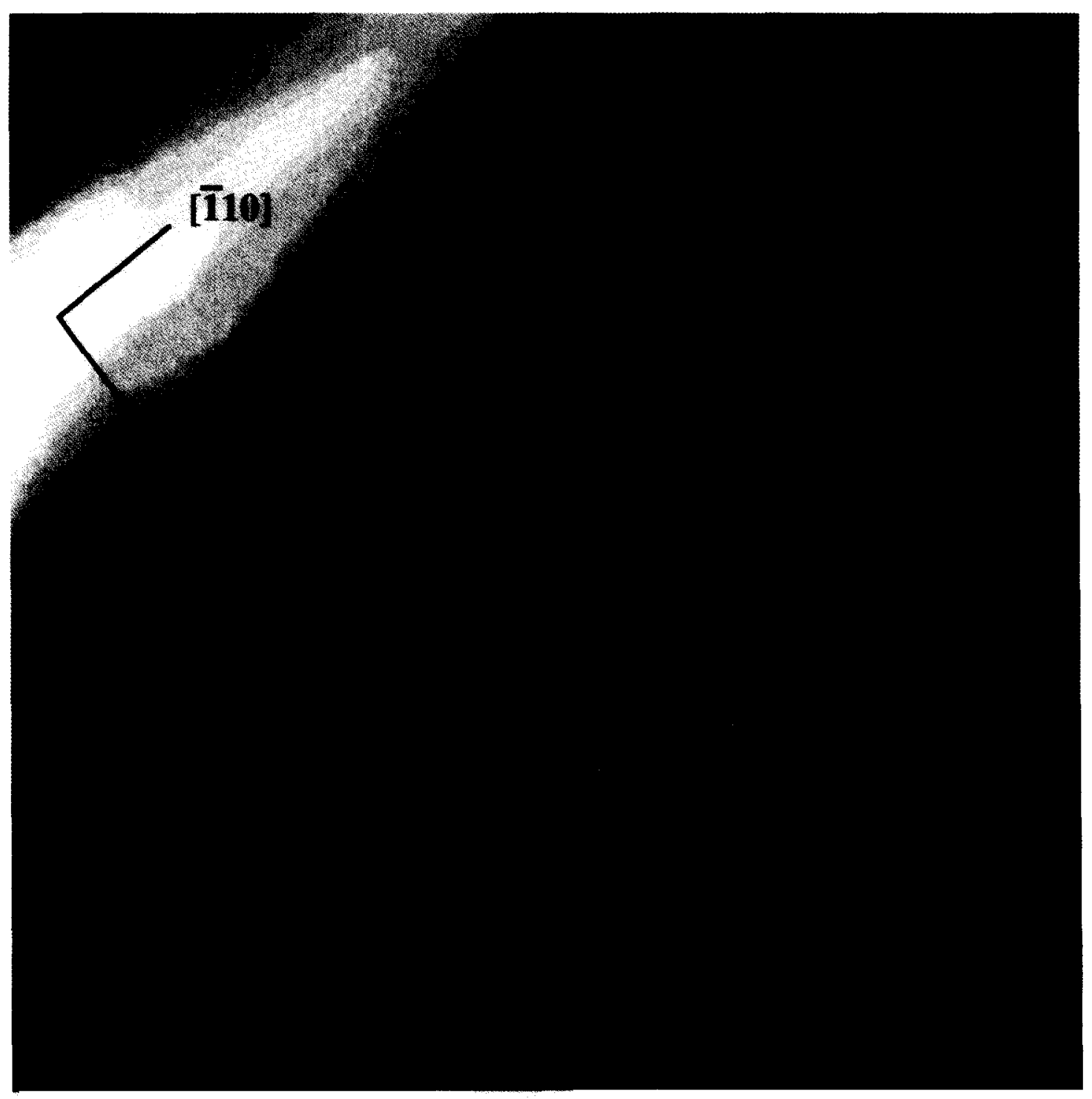

2000 Ang.

Fig. 2. GaAs surface after growth termination and recovery. 


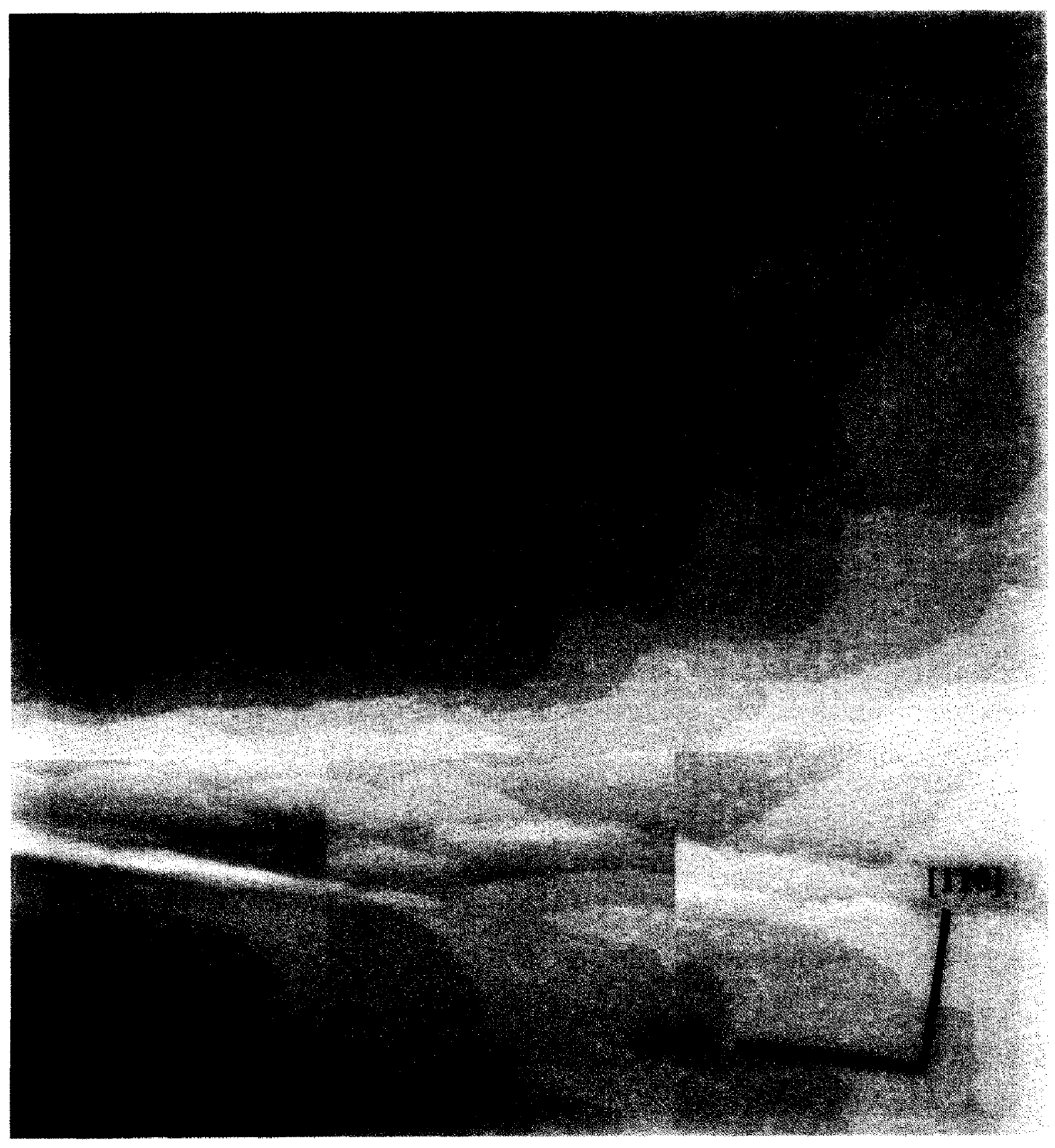

\section{Ang.}

Fig. 3. GaAs surface imaged after 0.25 layers deposited. 
With the onset of deposition there is a large drop in RHEED specular intensity. STM examination shows that a high density of small islands have nucleated on the large terraces of the recovered surface, fig. 3 . With $\sim 0.25$ monolayer deposited, the average island separation is $300 \AA$

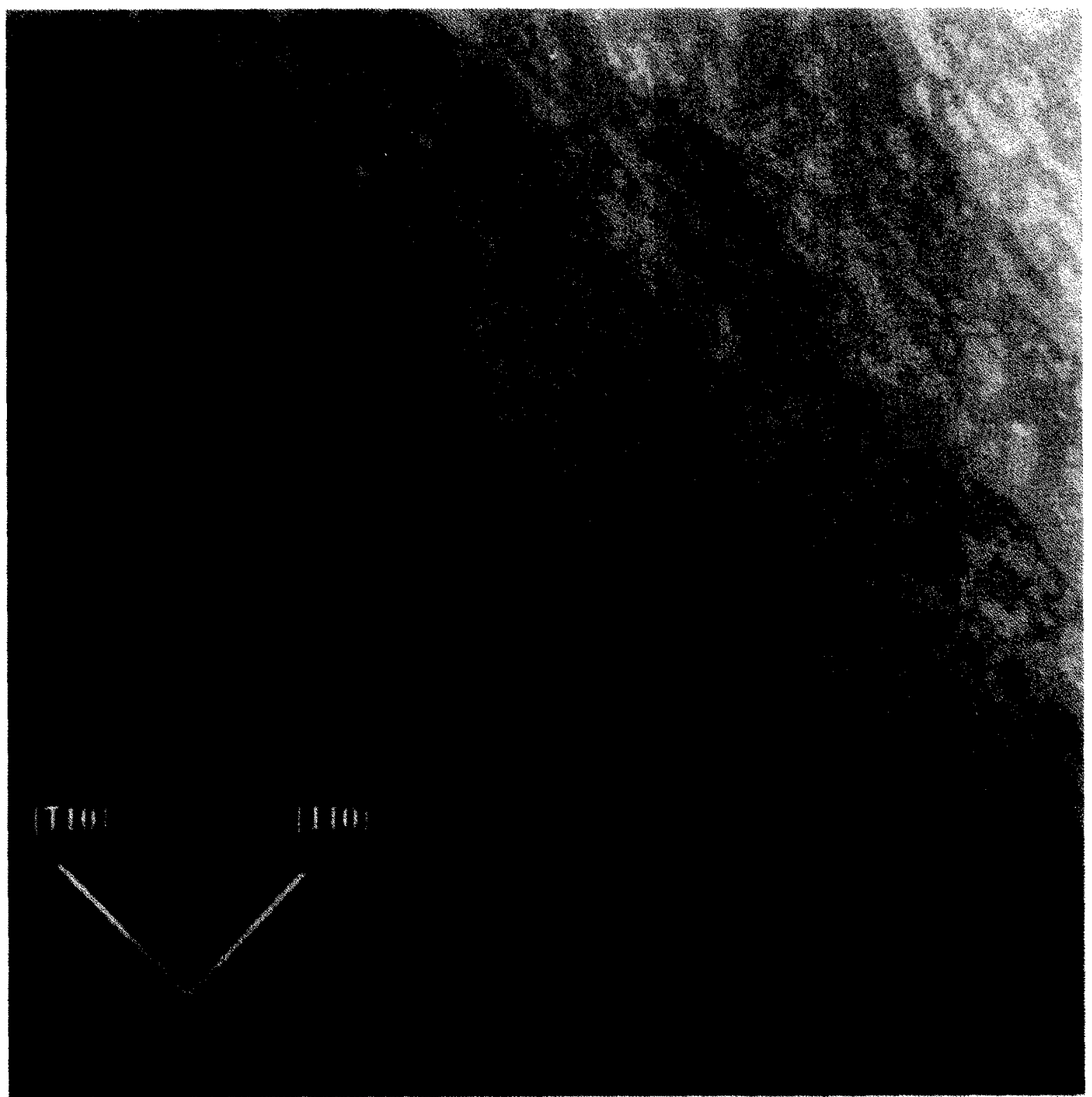

\section{Ang.}

Fig. 4. GaAs surface imaged after 3.5 layers deposited. 


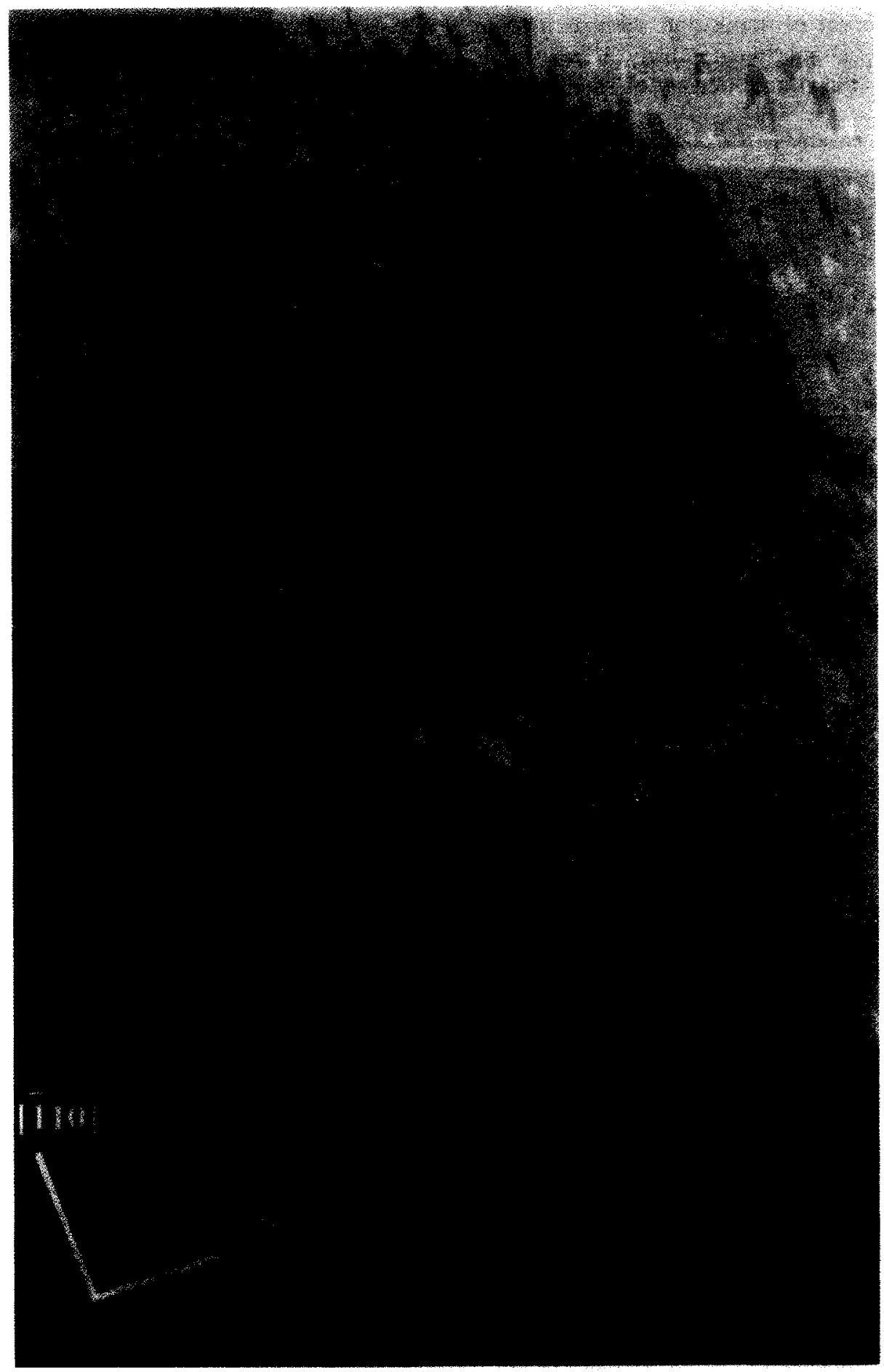

2000 Ang.

Fig. 5. GaAs surface imaged after 4.0 layers deposited. 


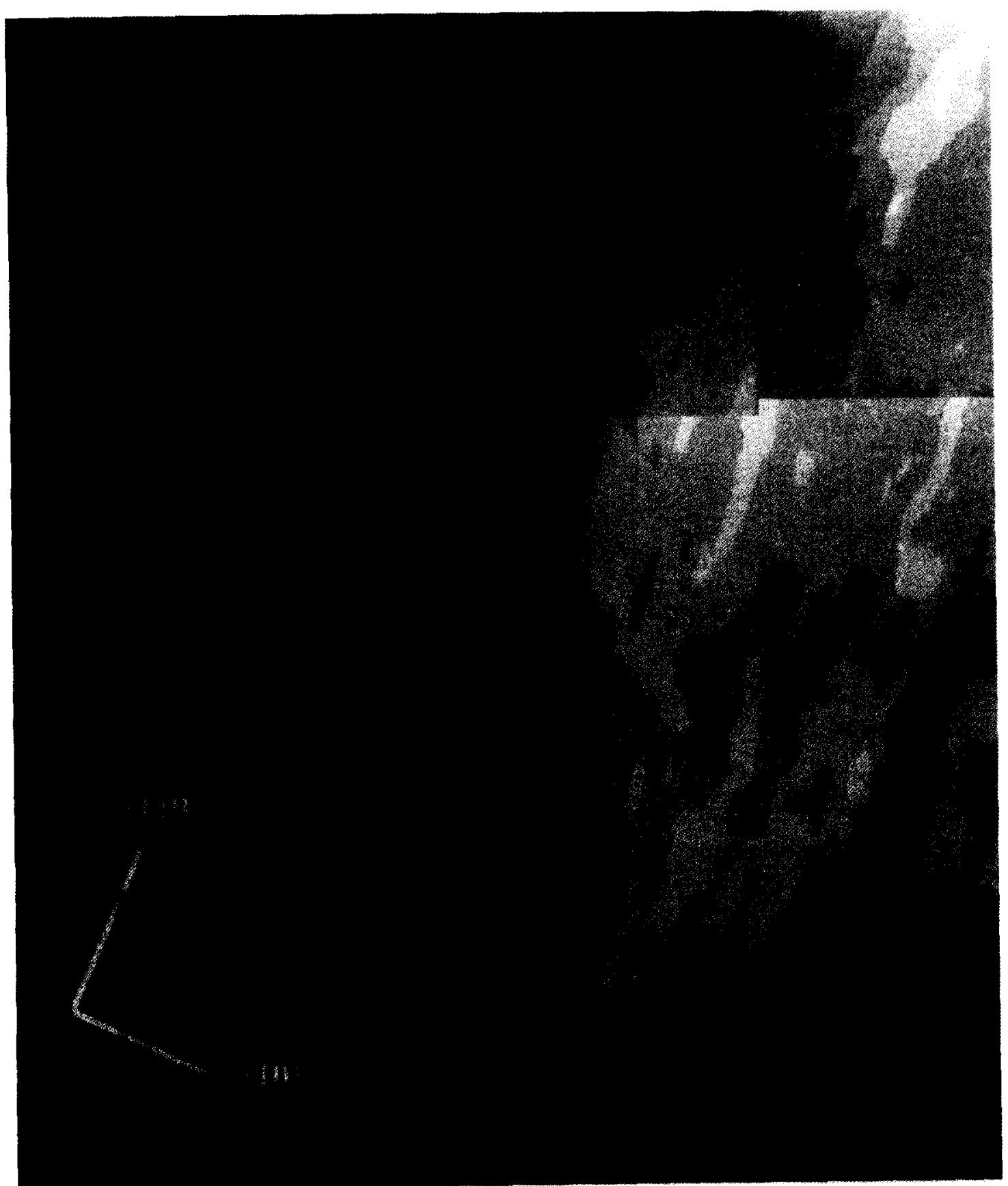

\section{Ang.}

Fig. 6. GaAs surface imaged after 60 layers deposited. 


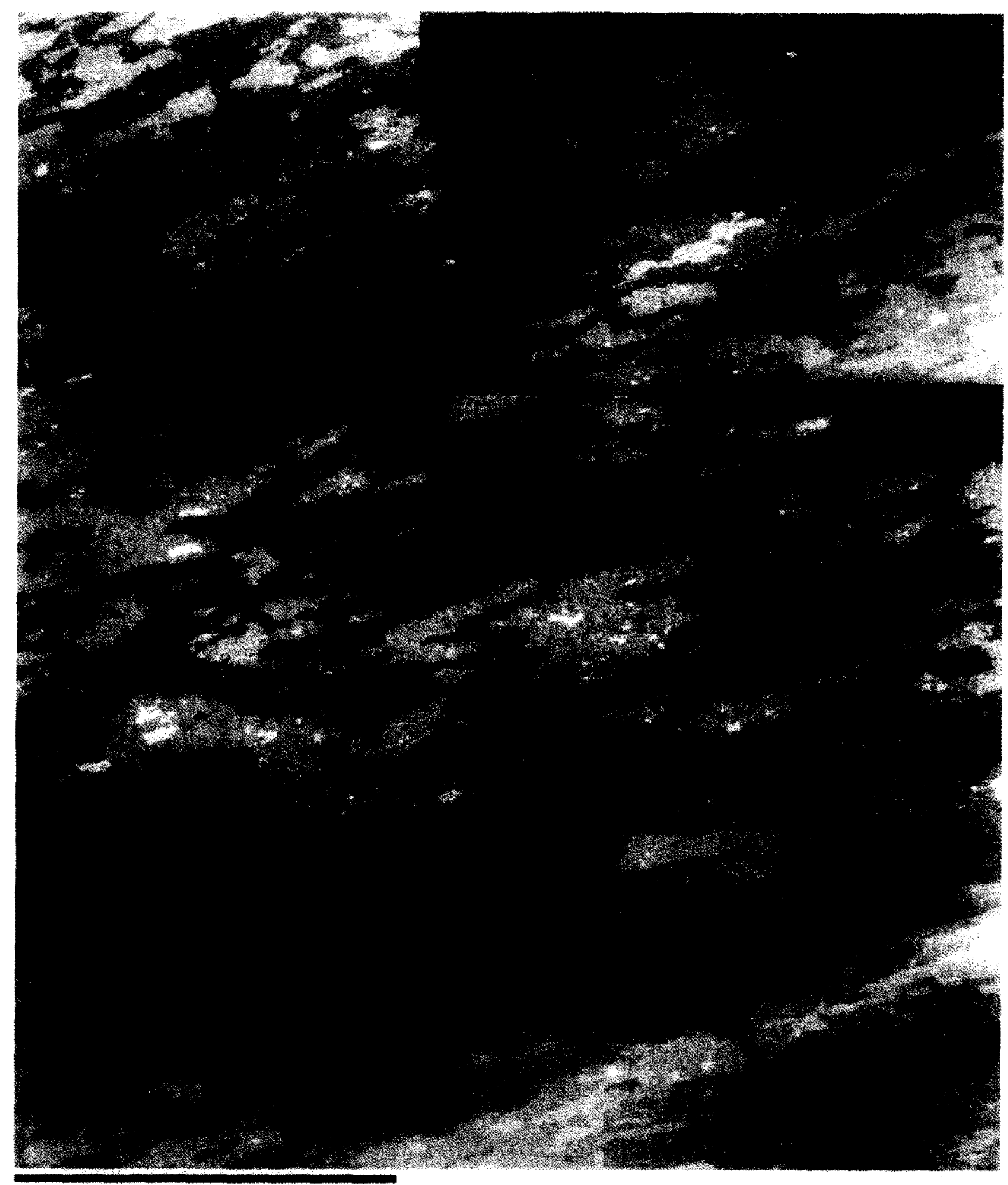

\section{Ang.}

Fig. 7. GaAs surface imaged after 120 layers deposited. 


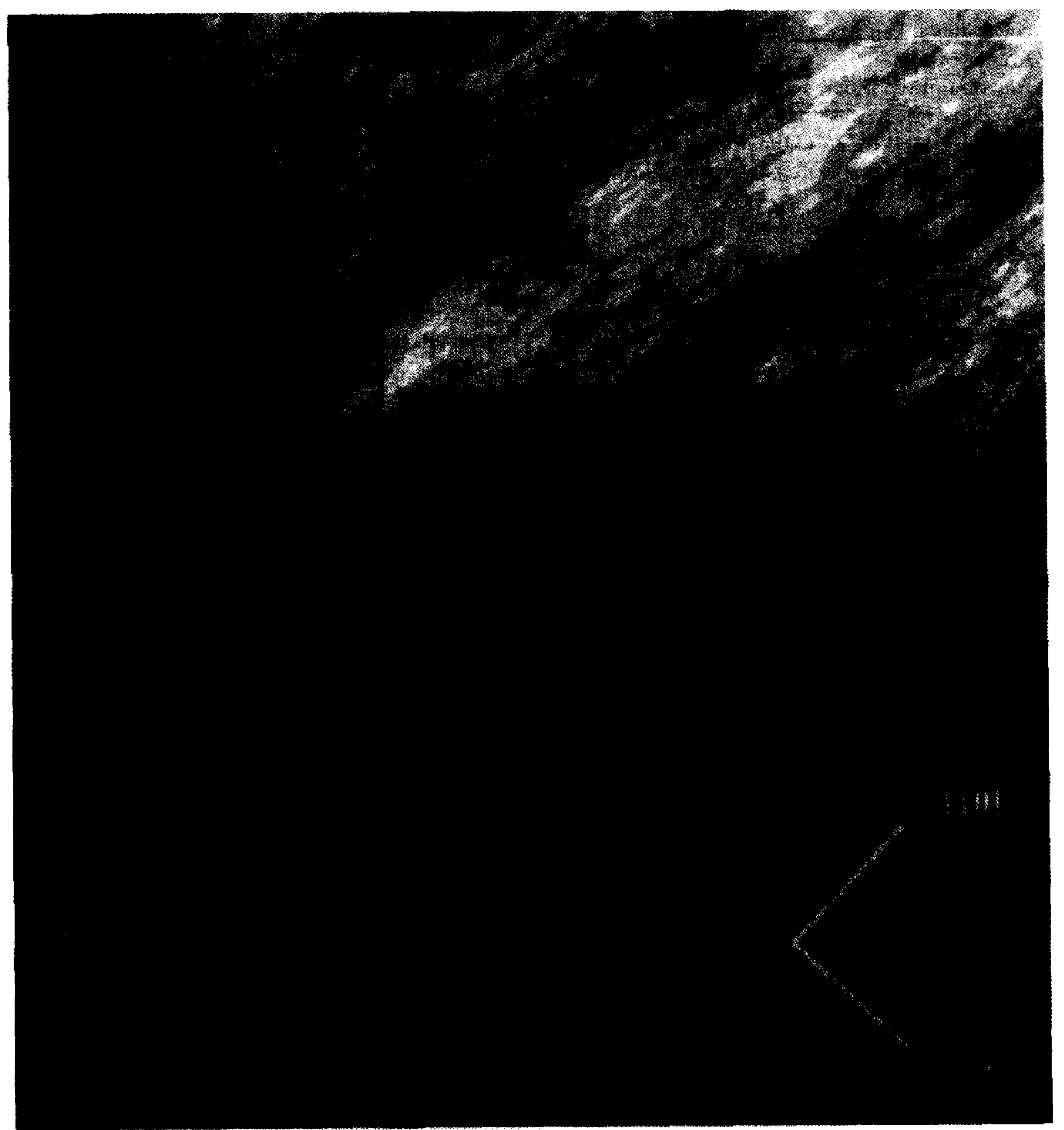

\section{Ang.}

Fig. 8. GaAs surface imaged after 1450 layers deposited. 
and the typical island is $80 \AA$ in diameter. The surface covered by these island is much less then the expected $25 \%$. It may be inferred that some growth has taken place at preexisting step edges. Growth of this nature would lead to an effective reduction of the flux responsible for island formation. This effect was first observed by RHEED and is detected by an anomalous increase in the period of the first intensity oscillation during growth [6].

With further deposition the RHEED intensity begins to oscillate. Figs. 4 and 5 show the surface as it appeared when the specularly scattered electron intensity was at a minimum and maximum, respectively. The most obvious difference in the images is the density of islands. At half monolayer coverage (fig. 4) the surface is observed to have many islands present. The island shapes vary greatly, however, they typically have about a 7-to-1 ratio in their length along the $[\overline{1} 10]$ direction versus the length along the [110] [7]. The nature of this anisotropy, whether diffusional and/or step edge energy, is difficult to precisely detcrmine and will be the subject of a future study. Turning to the image of the sample taken at monolayer completion, fig. 5, the surface is much smoother than at half coverage, although, not as smooth as the original starting surface. Both islands and holes, in approximately equal concentration, are present. These features are inevitable due to the stochastic nature of the deposition and diffusion processes involved during MBF growth.

The random build up of islands and holes on a terrace during growth gradually leads to a decrease in the temporal correlation between areas which are becoming smooth and areas which are becoming rough. The RHEED signature for this effect is a decay in the amplitude of specular intensity oscillations. Fig. 6 shows the GaAs surface when the RHEED oscillation amplitude has decayed to less than $5 \%$ of the original value. The surface is still quite smooth, with typically only two levels present within a $1500 \AA$ square area. The island shapes and terrace edges have become highly ramified. Features on the sample have coarsened to such a degree that it is difficult to distinguish the direction of the sample miscut.
During growth on this surface there is no cyclic variation in the step density and a steady or slowly varying state has been reached. Under these growth conditions the decay of the RHEED oscillations is not caused by an increase in interface roughness as had been previously postulated [8], but by the surface reaching a condition of constant step density $[9,10]$.

Upon further deposition the surface features continue to coarsen until they become so large that islands begin to nucleate on top of islands. At this stage the surface begins to roughen. Fig. 7 shows the surface at 120 monolayers. Four layers are now visible in a $1500 \AA$ square area. Even though the surface appears rough in these images the rms surface height fluctuation is only $2.5 \AA$. It is natural to expect this roughening to continue with further growth. However, we find that the surface evolves surprisingly little with the deposition of more material. Fig. 8 is an image of the surface after 1450 layers. The overall topography is essentially identical to that of the 120 layer film. This indicates that the system has reached a dynamical steady state [11].

In conclusion, we have presented large-scale images of the surface evolution of MBE grown GaAs. The initial growth proceeds by cyclic island formation and coalescence. This continues until a gradual coarsening of features allows the surface to reach a dynamical steady state. At all stages of growth the surface is smooth, with a maximum interface width of 4 monolayers. Upon growth interruption and surface recovery the terrace edges become straight and step bunching is found to occur.

\section{Acknowledgements}

We would like to thank Dr. D. Kessler, Dr. L. Sander, Dr. D. Vvedensky, Dr. A. Zangwill and Dr. B. Joyce for stimulating discussions. We are grateful to RHK technology and Burleigh Instruments for equipment grants. This work has been supported by the following grants: DAAL03-88G-0058, N00014-89-J-1519, NSF/DMR-8857828 and the Sloan Foundation. 


\section{References}

[1] General review of RHEED techniques and MBE can be found in: Reflection High Energy Electron Diffraction and Reflection Electron Imaging of Surfaces, NATO ASI Series B, Vol. 188, Eds. P.K. Larsen and P.J. Dobson (Plenum, New York, 1988).

[2] J.H. Neave, B.A. Joyce, P.J. Dobson and N. Norton, Appl. Phys. A 31 (1983) 1.

[3] B.G. Orr, C.W. Snyder and M. Johnson, Rev. Sci. Instrum. 6 (1991) 1400.

[4] M.D. Pashley, K.W. Haberern, W. Friday, J.M. Woodall and P.D. Kirchner, Phys. Rev. Lett. 60 (1988) 2176.

[5] T. Ide, A. Yamashita and T. Mizutani, Phys. Rev. B 46 (1992) 1905.
[6] D.G. Liu, C.P. Lee, K.H. Chang, J.S. Wu and D.C. Liou, Appl. Phys. Lett. 57 (1990) 1392;

T. Shitara, D.D. Vvedensky, M.R. Wildy, J. Zang, J.H. Neave and B.A. Joyce, Phys. Rev. B 46 (1992) 6815;

T. Shitara, PhD Thesis, Imperial College, University of London, 1992.

[7] G. Heller and M. Lagally, Appl. Phys. Lett. 60 (1992) 2675.

[8] H.C. Kang and J.W. Evans, Surf. Sci. 271 (1992) 321.

[9] J. Sudijono, M.D. Johnson, C.W. Snyder, M.B. Elowitz and B.G. Orr, Phys. Rev. Lett. 69 (1992) 2811.

[10] S. Clarke and D.D. Vvcdensky, Phys. Rev. Lett. 58 (1987) 2235.

[11] M.D. Johnson, J. Sudijono, C.W. Snyder, A. Hunt and B.G. Orr, to be published. 